\title{
Multiple Motion and Occlusion Segmentation with a Multiphase Level Set Method
}

\author{
Yonggang Shi, Janusz Konrad, W. Clem Karl \\ Department of Electrical and Computer Engineering \\ Boston University, Boston, MA 02215
}

\begin{abstract}
In this paper, we propose a new variational formulation for simultaneous multiple motion segmentation and occlusion detection in an image sequence. For the representation of segmented regions, we use the multiphase level set method proposed by Vese and Chan. This method allows an efficient representation of up to $2^{L}$ regions with $L$ level-set functions. Moreover, by construction, it enforces a domain partition with no gaps and overlaps. This is unlike previous variational approaches to multiple motion segmentation, where additional constraints were needed. The variational framework we propose can incorporate an arbitrary number of motion transformations as well as occlusion areas. In order to minimize the resulting energy, we developed a two-step algorithm. In the first step, we use a feature-based method to estimate the motions present in the image sequence. In the second step, based on the extracted motion information, we iteratively evolve all level set functions in the gradient descent direction to find the final segmentation. We have tested the above algorithm on both syntheticand natural-motion data with very promising results. We show here segmentation results for two real video sequences.
\end{abstract}

Keywords: Multiple Motion Segmentation, Occlusion, Multiphase level set method

\section{INTRODUCTION}

Motion segmentation is a challenging inverse problem in image sequence analysis. At the same time it is an important problem with applications in video compression (object-based coding, like MPEG-4), video processing (object-based frame rate conversion and deinterlacing), and computer vision (scene analysis, structure from motion). In this paper, we propose a new approach to multiple motion segmentation and occlusion detection that is based only on motion information and uses a variational multiphase level-set formulation.

Various approaches have been proposed to date to solve the problem of motion segmentation. Some methods are based on intensity analysis and use motion only as an additional cue during region merging or splitting, e.g.,. ${ }^{1,2}$ Based on evidence from previous work, ${ }^{3}$ we believe that the motion itself contains enough information to allow precise spatial segmentation of an image sequence.

Early spatial segmentation methods for video sequences suffered from lack of an explicit segmentation model. Later methods included such models by means of a probabilistic formulation (e.g., MAP) coupled with a particular random field model (e.g., Markov). Early MRF-based methods considered a planar scene composition only, ${ }^{4,5}$ while more sophisticated models were proposed later. ${ }^{6,7}$ The formalism of a MRF formulation of motion segmentation is often avoided by posing the problem directly in the energy minimization framework. For example, the energy minimization method developed by Mémin and Pérez ${ }^{8}$ uses active contours to describe regions and a multiresolution implementation to find the solution. Active contours have recently become a modeling/solution tool of choice for various segmentation problems, including motion-based. For example, works of Paragios and Deriche, ${ }^{9,10}$ Mansouri and Konrad, ${ }^{11,12}$ and Jehan-Besson et al. ${ }^{13,14}$ demonstrate various approaches to solving motion-based video segmentation using active-contour formulations.

The multiple motion and occlusion segmentation problem that we want to solve can be stated as follows. Given a pair of images from a video sequence at times $t_{k}$ and $t_{k+1}$, our goal is to segment the second image into regions of different motions by comparing it to the first image. We assume that motions present in the scene can

For further information the authors can be reached at: [yshi, jkonrad, wckarl] @bu.edu. 
be modeled as a finite number of transformations and the problem of motion segmentation is then to classify each pixel of the second image into one of the motion classes or as an occlusion.

We propose a variational method to solve this problem based on the multiphase level-set method developed recently by Vese and Chan. ${ }^{15}$ This method allows an efficient representation of up to $2^{L}$ regions with $L$ level-set functions. Moreover, by construction, it enforces a domain partition with no gaps and overlaps. This is unlike previous variational approaches to multiple motion segmentation, where additional constraints are needed. Mansouri and Konrad ${ }^{3}$ have proposed a system of multiple coupled level-set equations derived by extending minimization results for two motions. Despite additional constraints, gaps and overlaps can still occur in their results. More recently, Cramers ${ }^{16}$ proposed a motion segmentation approach based on the optical flow equation using the multiphase level-set method, but this method can only handle small motions and occlusions are not considered. Our approach is novel in several aspects. First, we propose a variational framework for the segmentation of multiple and general motions based on the multiphase level set method. Second, occlusions are modeled explicitly in our framework and can be detected together with regions of distinct motions.

The rest of the paper is organized as follows. In Section 2, we propose our variational framework based on the multiphase level-set method. Then, in Section 3 we develop a solution approach for the minimization of the proposed energy function. Promising experimental results on two real video sequences are presented in Section 4 , and conclusions are drawn in Section 5.

\section{VARIATIONAL FRAMEWORK}

We propose a general variational framework for simultaneous segmentation of multiple motions and occlusions based on the multiphase level set method of Chan and Vese. ${ }^{15}$

Let $f^{k}$ and $f^{k+1}$ denote two consecutive frames in an image sequence defined over a domain $\Omega$. Given a set of motion transformations $\mathbf{T}_{n}(n=1, \cdots, N)$, our goal is to segment the second frame $f^{k+1}$ into $N+1$ regions: $\Omega_{1}, \cdots, \Omega_{N}, \Omega_{N+1}$ such that $\Omega=\bigcup_{n=1}^{N+1} \Omega_{n}$. We assume that every motion transformation $\mathbf{T}_{n}$ maps region $\Omega_{n}$ in $f^{k+1}$ into a region in $f^{k}$, i.e., that $\mathbf{T}_{n}\left(\Omega_{n}\right) \subset \Omega$. The last region $\Omega_{N+1}$ represents occlusions, i.e., areas in $f^{k+1}$ that cannot be well matched in $f^{k}$.

Variational formulations and level-set solutions have recently become popular, and powerful techniques to solve various image segmentation problems. However, if a separate level set function is used to represent a specific class of regions, as is often the case, problems arise. It is not efficient and gaps/overlaps can occur between the zero level set of different functions. The gaps and overlaps can be controlled to some degree ${ }^{3}$ through more complicated implementation, but this is not always effective.

In this work, we use the multiphase level set method proposed by Chan and Vese ${ }^{15}$ to represent our segmentation. In the multiphase level set framework, $L$ level set functions $\phi_{1}, \cdots, \phi_{L}$ are used to partition the region $\Omega$ into up to $2^{L}$ regions as follows. For every pixel $x \in \Omega$, we assign to it a unique binary label $j=\left(H\left(\phi_{1}(x)\right), \cdots, H\left(\phi_{L}(x)\right)\right)$ where $H$ is the Heaviside function and $H\left(\phi_{i}(x)\right)(i=1, \cdots, L)$ is either 1 or 0 . Since the label $j$ can vary from 0 to $2^{L}-1$, the number of regions that can be represented is less than or equal to $2^{L}$. If we choose $L$ such that $N+1 \leq 2^{L}$, we can assign to each region $\Omega_{n}$ a binary label $j_{n}$. We define $\mathbf{I}=\left\{0,1, \cdots, 2^{L}-1\right\}$ to be the total index set induced by the $L$ level set functions, and $\mathbf{J}=\left\{j_{1}, \cdots, j_{N}, j_{N+1}\right\}$ to be the set of region labels used in our segmentation. The selection of region labels is arbitrary; they do not have to be in an increasing order as long as $\mathbf{J} \subseteq \mathbf{I}$ holds.

Considering the above multiphase representation, we propose a variational framework for the multiple motion and occlusion segmentation problem. We define the following energy function:

$$
\begin{aligned}
E= & \sum_{n=1}^{N} \int_{\Omega}\left|f^{k}\left(T_{n}(x)\right)-f^{k+1}(x)\right| \chi_{j_{n}}(x) d x+\sigma \int_{\Omega} \chi_{j_{N+1}}(x) d x \\
& +\gamma \sum_{n=N+2}^{2^{L}-1} \int_{\Omega} \chi_{j_{n}}(x) d x+\lambda \sum_{i=1}^{L} \int_{\Omega} \delta\left(\phi_{i}(x)\right)\left|\nabla \phi_{i}(x)\right| d x,
\end{aligned}
$$


where $\chi_{j_{n}}(x)$ is the indicator function for the region with label $j_{n}$. The moving region and occlusion labels are $j_{n} \in \mathbf{J}$ for $1 \leq n \leq N+1$, while unused labels are $j_{n} \in \mathbf{I} \backslash \mathbf{J}$ for $n>N+1$. The first term in the energy function above penalizes the matching error for each motion transformation $\mathbf{T}_{n}$. We choose the absolute intensity difference instead of the squared difference in order to measure the matching error as this leads to a smoother evolution speed for the level set function in our minimization process, and this, in turn, speeds up convergence. The second term is the cost assigned to the occluded region. It is an integration of a positive constant $\sigma$ over the region $\Omega_{N+1}$. Intuitively, this constant acts as a threshold and any pixel that cannot find a match in $f^{k}$ with an error less than $\sigma$ is classified into this region. The third term is an integration of a positive constant $\gamma$ over those regions that are not used in our segmentation. This term should be zero once the energy has been minimized. We achieve this by choosing $\gamma>\sigma$; a pixel will not be classified into these regions because it will always have a lower energy if it is classified into the region of occlusions. The last term is the sum of the length of all the zero-level sets and it is weighted by a positive parameter $\lambda$. By penalizing the length of zero-level sets, we can assure smooth boundaries.

As an example, if we have two motion transformations $\mathbf{T}_{1}$ and $\mathbf{T}_{2}$, we choose $L=2$ and select $j_{1}=(0,0), j_{2}=$ $(0,1), j_{3}=(1,1), j_{4}=(1,0)$. The energy function is then defined as follows:

$$
\begin{aligned}
E= & \int_{\Omega}\left|f^{k}\left(T_{1}(x)\right)-f^{k+1}(x)\right|\left(1-H\left(\phi_{1}(x)\right)\right)\left(1-H\left(\phi_{2}(x)\right)\right) d x+ \\
& \int_{\Omega}\left|f^{k}\left(T_{2}(x)\right)-f^{k+1}(x)\right|\left(1-H\left(\phi_{1}(x)\right)\right) H\left(\phi_{2}(x)\right) d x+ \\
& \sigma \int_{\Omega} H\left(\phi_{1}(x)\right) H\left(\phi_{2}(x)\right) d x+\gamma \int_{\Omega} H\left(\phi_{1}(x)\right)\left(1-H\left(\phi_{2}(x)\right)\right) d x+ \\
& \lambda\left(\int_{\Omega} \delta\left(\phi_{1}(x)\right)\left|\nabla \phi_{1}(x)\right| d x+\int_{\Omega} \delta\left(\phi_{2}(x)\right)\left|\nabla \phi_{2}(x)\right| d x\right)
\end{aligned}
$$

\section{ENERGY MINIMIZATION}

We minimize the energy function in (1) with a two-step algorithm. In the first step, we estimate all the motion transformations that are present between the two frames. Then, we used a level set method to evolve all the level-set functions to achieve the final segmentation.

We assume for now that all motion transformations $\mathbf{T}$ can be modeled as affine mappings. We perform motion classification and estimation using a feature-based method developed by Mansouri and Konrad. ${ }^{3}$ In this method, we first extract feature points from both images $f^{k}$ and $f^{k+1}$ using the algorithm proposed by Harris, ${ }^{17}$ and then we establish correspondences between feature points by maximizing normalized cross-correlation over a square block. We finally use the K-means algorithm to classify and estimate the set of affine motions $\left\{\mathbf{T}_{1}, \cdots, \mathbf{T}_{N}\right\}$ that are present in the set of feature points.

In the second stage of our method, we use the results from the above step as inputs and perform motion and occlusion segmentation using the multiphase level set method. Since all the motion transformations have been estimated, we can compute the first variation of the energy with respect to the $L$ level-set functions and minimize the energy by evolving them in the gradient descent direction with the numerical method proposed by Osher and Sethian. ${ }^{18}$ We denote the binary label for each region as $j_{n}=\left(a_{n}^{1}, \cdots, a_{n}^{L}\right)$ for $1 \leq n \leq 2^{L}-1$. With this notation the indicator function of each region can be expressed as:

$$
\chi_{j_{n}}(x)=\prod_{i=1}^{L}\left[\left(1-a_{n}^{i}\right)+\left(2 a_{n}^{i}-1\right) H\left(\phi_{i}(x)\right)\right] .
$$

The dynamic evolution equation for level-set function $\phi_{l}(l=1, \cdots, L)$ in the gradient descent direction of the energy is then:

$$
\frac{d \phi_{l}(x)}{d t}=\delta\left(\phi_{l}(x)\right)\left\{\sum_{n=1}^{2^{L}-1}\left[g_{n}(x)\left(1-2 a_{n}^{l}\right) \prod_{i=1, i \neq l}^{L}\left(\left(1-a_{n}^{i}\right)+\left(2 a_{n}^{i}-1\right) H\left(\phi_{i}(x)\right)\right)\right]+\lambda \nabla \cdot \frac{\nabla \phi_{l}(x)}{\left|\nabla \phi_{l}(x)\right|}\right\}
$$


where the function $g_{n}$ is defined as follows:

$$
g_{n}(x)= \begin{cases}\left|f^{k}\left(T_{n}(x)\right)-f^{k+1}(x)\right| & \text { if } 1 \leq n \leq N \\ \sigma & \text { if } n=N+1 \\ \gamma & \text { if } n \geq N+2\end{cases}
$$

The second term $\nabla \cdot \frac{\nabla \phi_{l}(x)}{\left|\nabla \phi_{l}(x)\right|}$ in (3) is the curvature of the level sets of $\phi_{l}$. It enforces smoothness of boundaries.

For example, the evolution equations of $\phi_{1}$ and $\phi_{2}$ resulting from minimization of the energy in (2) is:

$$
\begin{aligned}
\frac{d \phi_{1}(x)}{d t}= & \delta\left(\phi_{1}(x)\right)\left[\left(\sigma-\left|f^{k}\left(T_{2}(x)\right)-f^{k+1}(x)\right|\right) H\left(\phi_{2}(x)\right)\right. \\
& \left.+\left(\gamma-\left|f^{k}\left(T_{1}(x)\right)-f^{k+1}(x)\right|\right)\left(1-H\left(\phi_{2}(x)\right)\right)+\lambda \nabla \cdot \frac{\nabla \phi_{1}(x)}{\left|\nabla \phi_{1}(x)\right|}\right] \\
\frac{d \phi_{2}(x)}{d t}= & \delta\left(\phi_{2}(x)\right)\left[(\sigma-\gamma) H\left(\phi_{1}(x)\right)\right. \\
& \left.+\left(\left|f^{k}\left(T_{2}(x)\right)-f^{k+1}(x)\right|-\left|f^{k}\left(T_{1}(x)\right)-f^{k+1}(x)\right|\right)\left(1-H\left(\phi_{1}(x)\right)\right)+\lambda \nabla \cdot \frac{\nabla \phi_{2}(x)}{\left|\nabla \phi_{2}(x)\right|}\right]
\end{aligned}
$$

For numerical implementations of these partial differential equations, we use the numerical schemes of level set methods. ${ }^{15,18}$

\section{EXPERIMENTAL RESULTS}

We present some preliminary segmentation results of our method on two real video sequences.

We first test our method on a video sequence where only a car is moving against a stationary background. Thus, there are three regions to be segmented: the moving car, the static background and the occluded region. A pair of frames from this video sequence are shown in Fig. 1. In the first step of our method, we estimated motion occurring between the two frames using feature correspondences. The feature points detected are shown in Figure 2.

In the second step, we use the multiphase level-set method to segment the second frame $f^{k+1}$. We use two level-set functions $\phi_{1}$ and $\phi_{2}$ for this example. Their zero-level sets are plotted in Fig. 3(a). We choose the initial zero-level set of each function to be composed of many small circles that are spread over the whole image domain. This lets us avoid local minima and has proven useful in our experiments. The level-set function is chosen as the signed distance function of these small circles. Then, we evolve these two level-set functions iteratively by solving the time-dependent partial differential equation (3). The final segmentation results $\left(\phi_{1}\right.$ and $\left.\phi_{2}\right)$ are shown in Fig. 3(b). The zero-level sets of the level-set functions are plotted separately as black and white contours. Since the visibility of the zero-level sets in this figure is limited due to the background texture, we also present our segmentation results in a multiphase representation in Fig. 4. In this figure, every region is denoted by its binary label induced from $\phi_{1}$ and $\phi_{2}$, and the corresponding texture is overlaid on it. Region $(0,0)$ represents the occluded region in $f^{k+1}$ that is not present in $f^{k}$. Region $(0,1)$ represents the moving car, and region $(1,1)$ is the static background. Because in this sequence only 3 regions are associated with meaningful objects, we assigned the cost $\gamma$ to region $(1,0)$ in our energy function. The results show that our method eliminated this region although it was present at the start of the iterations. The rear of the car, parts of the pavement and parts of the two cars parked behind the moving car are not visible in $f^{k}$, and thus are correctly estimated as occluded areas. The two small patches, one above the other, result from occlusion by the white pole. The small patch on the left is due to the occlusion of the background, visible through the window, by the moving car's frame. Although the moving car is fairly accurately estimated, some car areas are missing, classified as the occluded region or the static background. There are two reasons for this. First, some of those areas really correspond to features one can see through the window of the car; they do not have a good match after the car has moved. The second reason is spatial variation of illumination that makes part of car's door match some other part of the car instead of its corresponding position in the previous frame. 
The second video sequence we tested is from web site http://i21www.ira.uka.de/image_sequences. Two frames to be segmented are shown in Fig. 5. Features detected for these two images are shown in Fig. 6. The results of our first step is that we have two moving objects and a static background. Thus, overall four regions need to be found: three regions of distinct motions and one occluded region. We use two level set functions $\phi_{1}$ and $\phi_{2}$ here. Clearly, no unassigned region will result. As before, we choose small circles for initialization as shown in Fig. 7(a). The final segmentation results are shown in Fig. 7(b). A multiphase representation of the segmentation results for this example is presented in Fig. 8. Region $(0,0)$, representing occlusions, correctly marks part of the pavement with the white arrow that was covered by the white car in the first frame. Region $(0,1)$ represents the first moving object, i.e., regions with the best match using the same motion transformation as that of the white car. Region $(1,0)$ is the result corresponding to the motion transformation of the black car. Region $(1,1)$ is the static background. Interestingly not all occlusions are captured by region $(0,0)$. This is not surprising where intensity is uniform and matches very well other parts of the road using the motion transformations of either car. It is somewhat unexpected, however, that the white line above the black car whose part is occluded in the first frame did not get detected (perhaps because only a small part was occluded). Also, as we can see from the results for region $(0,1)$ and $(1,0)$, shades of both cars are segmented correctly together with the cars. This example demonstrates that our method can segment multiple motions and occlusions successfully.

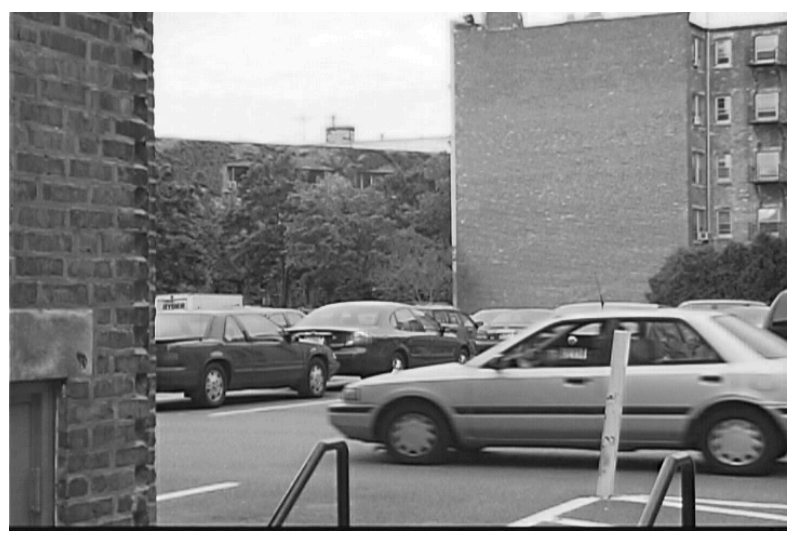

(a)

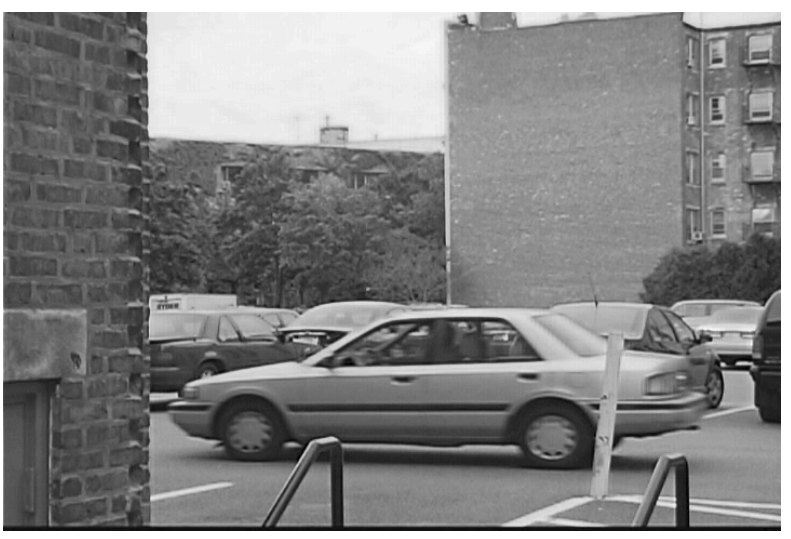

(b)

Figure 1. Two frames of the first video sequence: (a) first frame $f^{k}$; (b) second frame $f^{k+1}$.

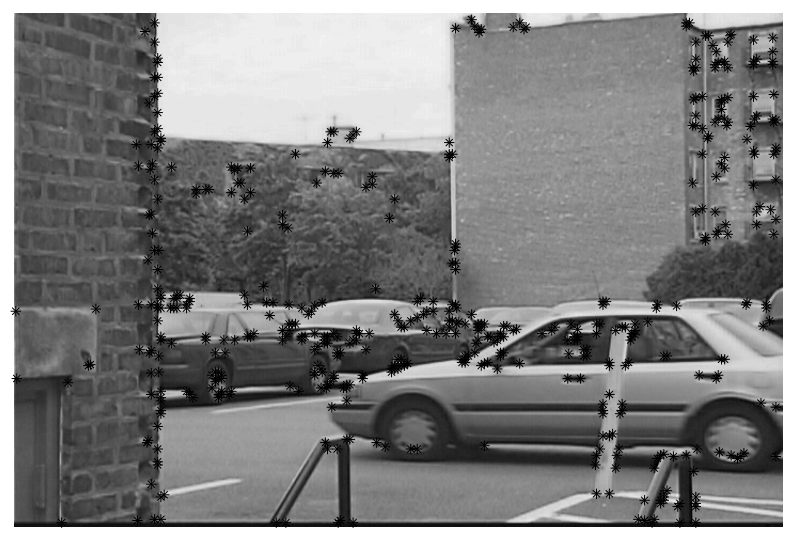

(a)

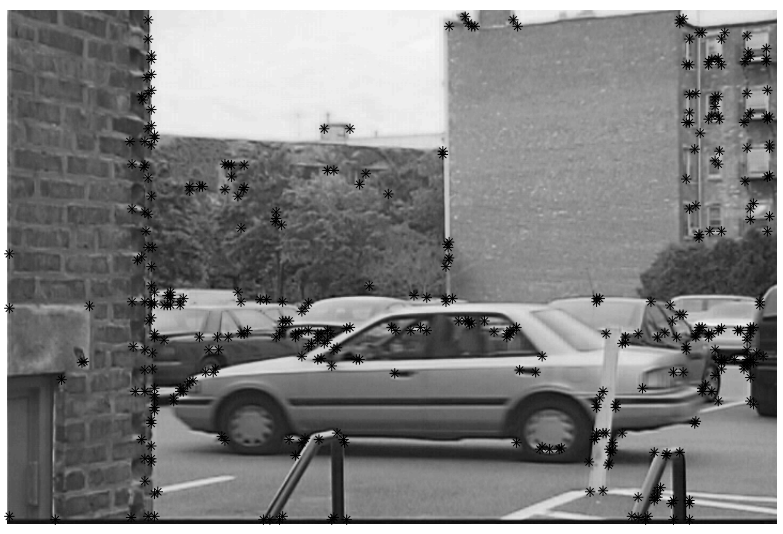

(b)

Figure 2. Feature points detected in the first video sequence using Harris's ${ }^{17}$ method (every feature point is represented by a star in the picture). (a) Results for the first frame $f^{k}$; (b) results for the second frame $f^{k+1}$. 


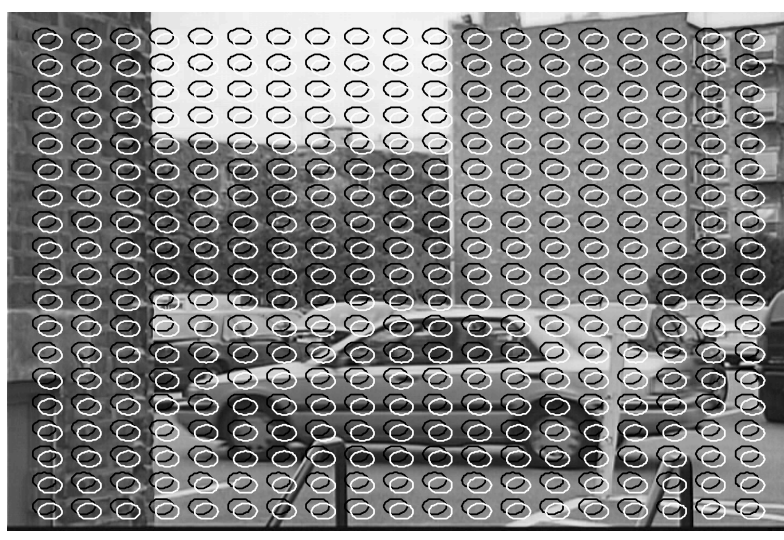

(a)

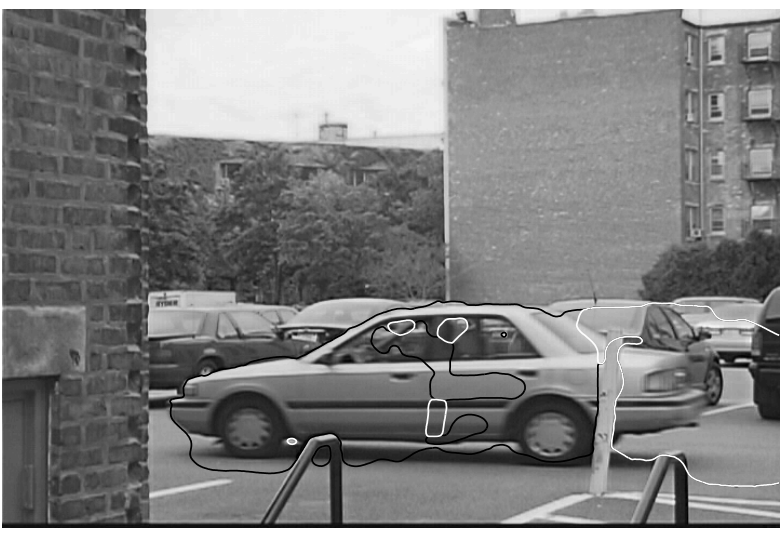

(b)

Figure 3. (a) Initial zero-level sets of $\phi_{1}$ (black) and $\phi_{2}$ (white) for the first example; (b) final segmentation results (zero-level sets) of $\phi_{1}$ (black) and $\phi_{2}$ (white) for the first example.

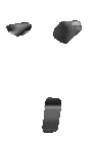

(a)

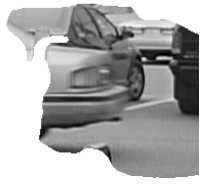

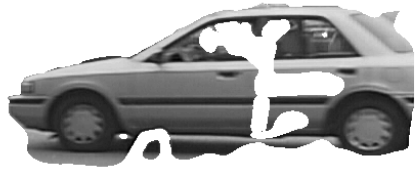

(b)

(c)

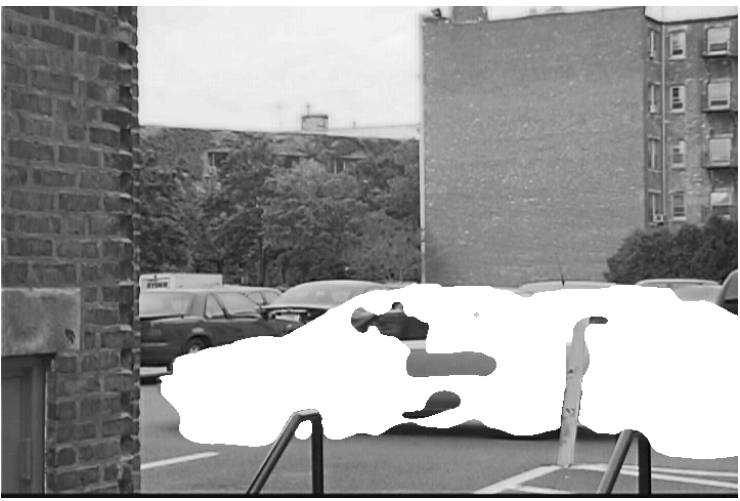

$(\mathrm{d})$

Figure 4. Multiphase representation of the segmentation results for the first example. (a) region $(0,0)$ - occlusions; (b) region $(0,1)$ - moving car; (c) region $(1,0)$ - not used; (d) region $(1,1)$ - static background. 


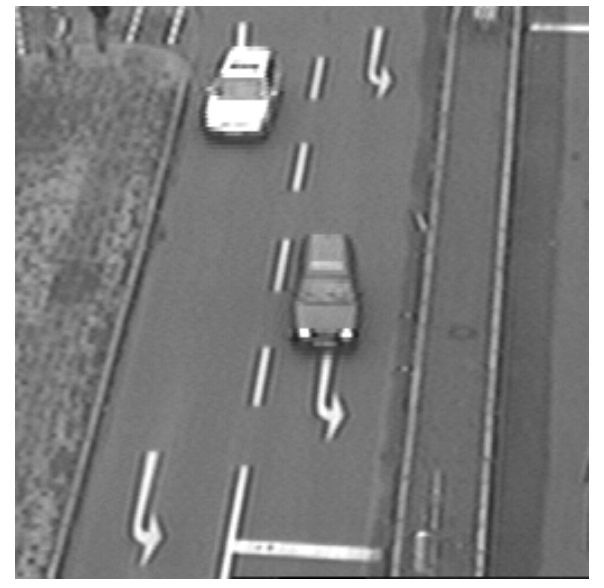

(a)

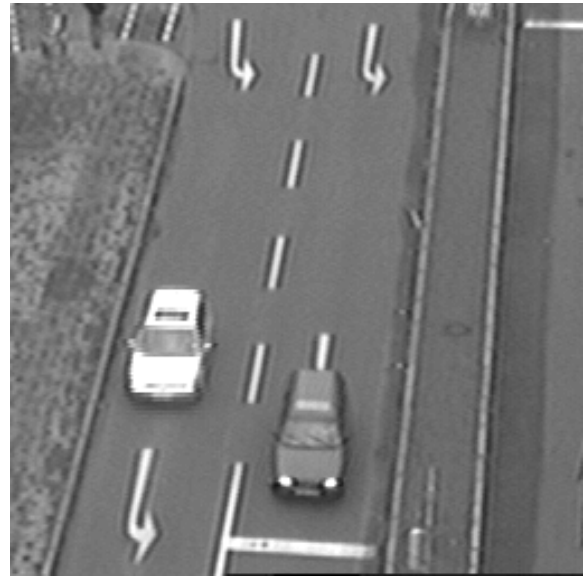

(b)

Figure 5. Two frames from the second video sequence: (a) first frame $f^{k}$; (b) second frame $f^{k+1}$.

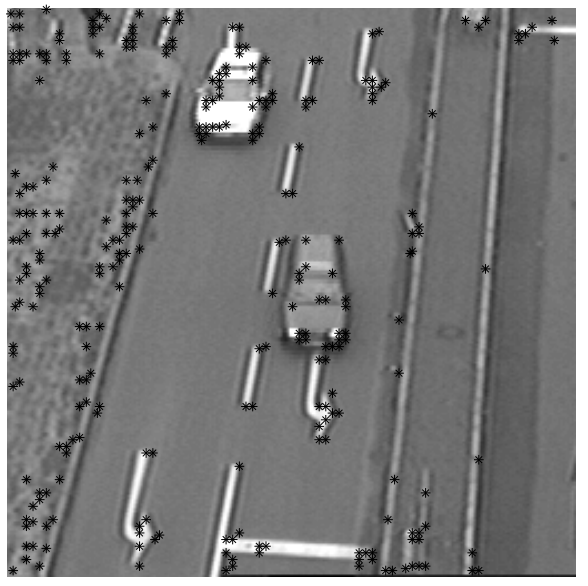

(a)

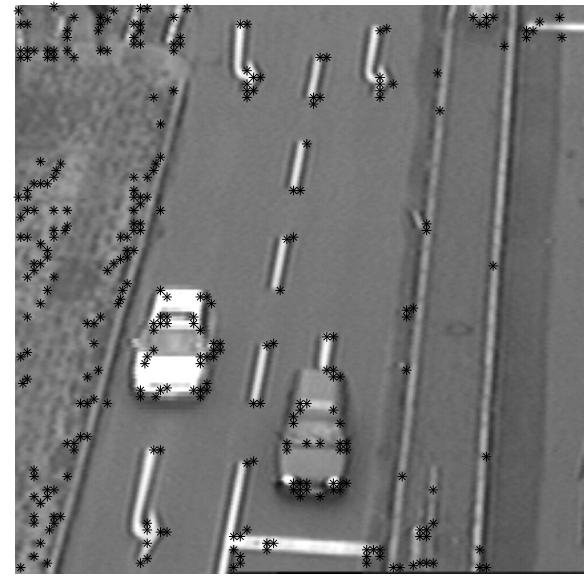

(b)

Figure 6. Feature points detected in the second video sequence using Harris's ${ }^{17}$ method (every feature point is represented by a star in the picture). (a) Results for the first frame $f^{k}$; (b)Results for the second frame $f^{k+1}$. 


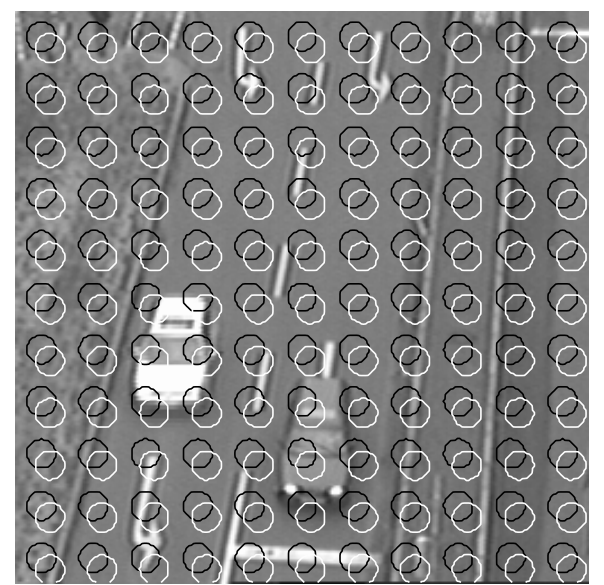

(a)

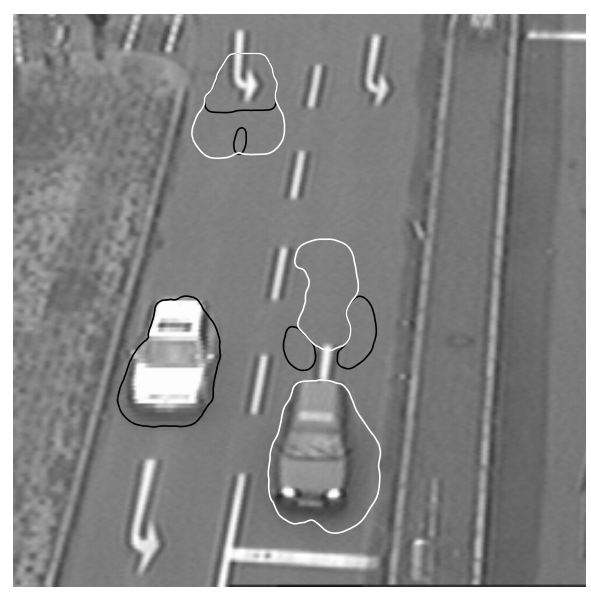

(b)

Figure 7. (a) Initial zero-level sets of $\phi_{1}$ (black) and $\phi_{2}$ (white) for the second example; (b) final segmentation results (zero-level sets) of $\phi_{1}$ (black) and $\phi_{2}$ (white) for the second example.
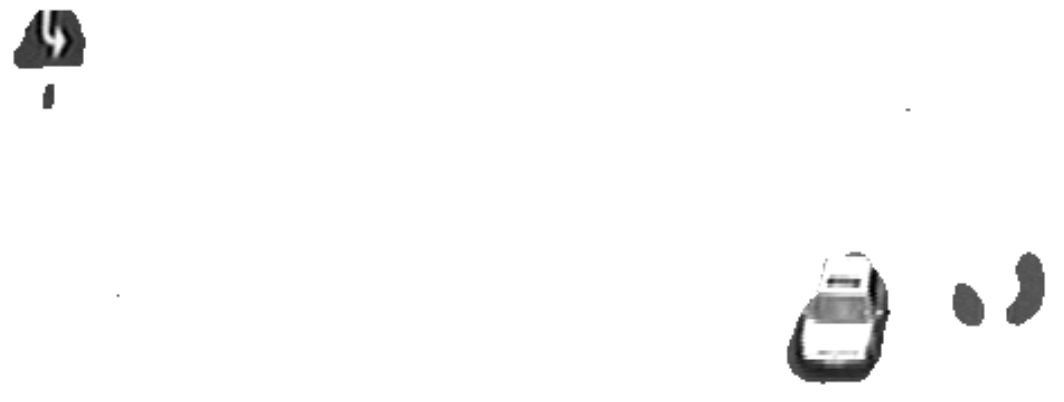

(a)

(b)

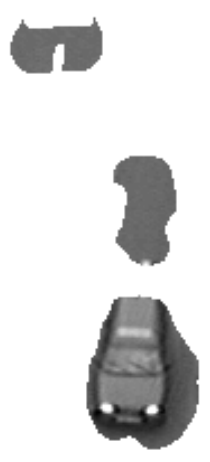

(c)

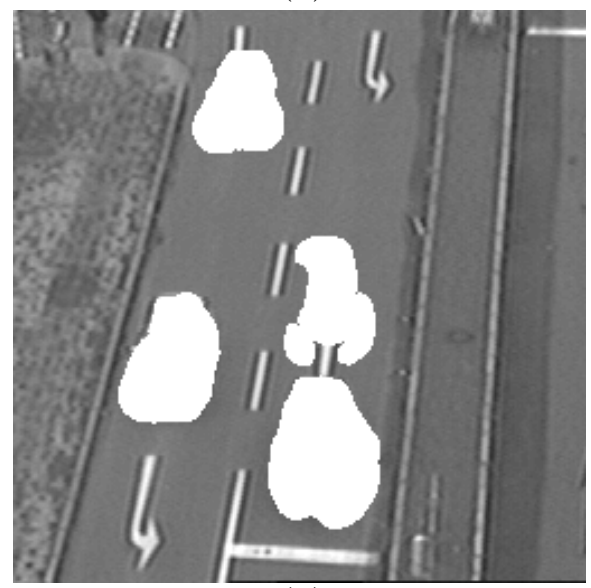

(d)

Figure 8. Multiphase representation of the segmentation results for the second example. (a) region $(0,0)$ - occlusions; (b) region $(0,1)$ - moving white car; (c) region $(1,0)$ - moving black car; (d) region $(1,1)$ - static background. 


\section{CONCLUSIONS}

In this paper, we presented a general variational framework for the problem of multiple motion and occlusion segmentation in a sequence of moving images. In our framework, only motion information is used, but the incorporation of other cues, such as gradient information, is straightforward. One important feature of our method is that it is based on the multiphase level set method, thus an arbitrary number of general motions can be incorporated into the framework easily. For future research, the application of our method to more complicated video sequences will be investigated.

\section{ACKNOWLEDGMENTS}

The work of the first and third author was partially supported by the Engineering Research Centers Program of the National Science Foundation under award number EEC-9986821, National Institutes of Health under Grant NINDS 1 R01 NS34189, and Air Force under award number F49620-03-1-0257. The work of the second author was supported by the National Science Foundation under grant number CCR-0209055.

\section{REFERENCES}

1. N. Diehl, "Object-oriented motion estimation and segmentation in image sequences," Signal Process. Image Commun. 3, pp. 23-56, Feb. 1991.

2. F. Dufaux, F. Moscheni, and A. Lippman, "Spatio-temporal segmentation based on motion and static segmentation," in Proc. IEEE. Int. Conf. Image Processing, 1, pp. 306-309, Oct. 1995.

3. A. Mansouri and J. Konrad, "Multiple motion segmentation with level sets," IEEE Trans. Image Process. 12, pp. 201-220, Feb. 2003.

4. B. Buxton and D. Murray, "Optical flow segmentation as an ill-posed and maximum likelihood problem," Image Vis. Comput. 3(4), pp. 163-169, 1985.

5. D. Murray and B. Buxton, "Scene segmentation from visual motion using global optimization," IEEE Trans. Pattern Anal. Machine Intell. 9, pp. 220-228, Mar. 1987.

6. C. Stiller, "Object-based estimation of dense motion fields," IEEE Trans. Image Process. 6, pp. 234-250, Feb. 1997.

7. M. Chang, A. Tekalp, and M. Sezan, "Simultaneous motion estimation and segmentation," IEEE Trans. Image Process. 6, pp. 1326-1333, Sept. 1997.

8. E. Mémin and P. Pérez, "Dense estimation and object-based segmentation of the optical flow with robust techniques," IEEE Trans. Image Process. 7, pp. 703-719, May 1998.

9. N. Paragios and R. Deriche, "Geodesic active regions for motion estimation and tracking," in Proc. IEEE Int. Conf. Computer Vision, pp. 688-694, Sept. 1999.

10. N. Paragios and R. Deriche, "Geodesic active contours and level sets for the detection and tracking of moving objects," IEEE Trans. Pattern Anal. Machine Intell. 22, pp. 266-280, Mar. 2000.

11. A.-R.Mansouri and J. Konrad, "Motion segmentation with level sets," in Proc. IEEE Int. Conf. Image Processing, 2, pp. 126-130, Oct. 1999.

12. A.-R.Mansouri and J. Konrad, "Minimum description length region tracking with level sets," in Proc. SPIE Image and Video Communications and Process, 3974, pp. 515-525, Jan. 2000.

13. S. Jehan-Besson, M. Barlaud, and G. Aubert, "Detection and tracking of moving objects using a new level set based method," in Proc. Int. Conf. Patt. Recog., pp. 1112-1117, Sept. 2000.

14. S. Jehan-Besson, M. Barlaud, and G. Aubert, "An object-based motion method for video coding," in Proc. IEEE Int. Conf. Image Processing, pp. 502-505, 2001.

15. L. Vese and T. Chan, "A multiphase level set framework for image segmentation using the mumford and shah model," International Journal of Computer Vision 50(3), pp. 271-293, 2002.

16. D. Cremers, "A multiphase level set framework for motion segmentation," in 4th Int. Conf. on Scale Space Theories in Computer Vision, L. Griffin and M. Lillholm, eds., Springer, (Isle of Skye, Scotland), Jun. 2003.

17. C. Harris and M. Stephens, "A combined corner and edge detector," in Alevy Vision Conference, pp. 147$151,1988$.

18. S. Osher and J. Sethian, "Fronts propagation with curvature-dependent speed: algorithms based on Hamilton-Jacobi formulations," Journal of computational physics 79, pp. 12-49, 1988. 